\title{
Repeat Expansions in Leukoencephalopathy
}

Adult onset leukoencephalopathy is a heterogeneous condition that often poses a diagnostic challenge. After infectious, inflammatory, neoplastic, drug-induced and acquired demyelinating disorders have been excluded, the range of potential genetic causes is daunting. Even in this era of next-generation sequencing and targeted disease gene panels, the precise genetic diagnosis often remains elusive $(1,2)$. In this issue, Okubo and colleagues (3) report that a recently identified GGC repeat expansion in NOTCH2NLC (also known as NBPF19) may be a relatively common genetic cause of adult onset leukoencephalopathy. This newest repeat expansion disease also points to an exciting realization: a cluster of formerly unlinked disorders may well constitute a spectrum of age-related neurodegenerative diseases with shared clinical, radiographic, neuropathological and molecular features.

The current report builds on the recent discovery that a GGC repeat expansion in NOTCH2NLC (4-7) is the major cause of neuronal intranuclear inclusion disease (NIID), a fatal neurodegenerative disorder that also involves peripheral organs. In patients, the diagnosis of NIID is made via skin biopsy which reveals the neuropathological hallmark of disease, ubiquitinpositive intranuclear inclusions that occur in many organs and cell types, including neurons and glia of the central and peripheral nervous systems. Before skin biopsy became part of the workup, most cases of NIID were only discovered when brain autopsy revealed the characteristic neuropathological finding. While NIID often runs in families with an autosomal dominant pattern of inheritance, sporadic disease in older persons is not infrequent. Clinical features of NIID vary greatly, with younger onset patients usually presenting with limb weakness and older onset patients with dementia (8). Other common findings include neuropathy, movement disorder (most often ataxia) and signs of autonomic dysfunction, as well as white matter signal abnormalities on brain MRI. In particular, high signal intensity at the corticomedullary junction in diffusion-weighted imaging is nearly always found (8).

Given the white matter involvement in NIID, Okubo and colleagues asked whether this newly discovered GCC expansion might also be a cause of adult onset leukoencephalopathy in which other genetic causes had been excluded. They evaluated 101 Japanese patients, in 51 of whom a custom disease gene panel (1) for causes of leukoencephalopathy had proven negative; the remaining 50 newly recruited patients were first assessed by whole exome sequencing, which uncovered 7 pathogenic mutations, 6 of which were in the CADASIL disease gene, NOTCH3. (A notable incidental finding of this study is that mutations in NOTCH3 can manifest as adult onset leukoencephalopathy lacking the typical characteristics of CADASIL.) Remarkably, repeatprimed PCR analysis in the remaining 94 patients identified the NIID-associated GGC repeat expansion in 12 patients, making it perhaps the most common identified cause of adult onset leukoencephalopathy.

What does this collection of a dozen Japanese patients tell us about the characteristics of NOTCH2NLC repeat expansion-mediated leukoencephalopathy? Symptom onset ranged from ages 27 to 70 and nearly all patients manifested dementia, decreased deep tendon reflexes,

This is the author manuscript accepted for publication and has undergone full peer review but has not been through the copyediting, typesetting, pagination and proofreading process, which may lead to differences between this version and the Version of Record. Please cite this article as doi: 10.1002/ana.25613

This article is protected by copyright. All rights reserved. 
neuropathy and autonomic dysfunction. A minority developed tremor or experienced encephalitic episodes. The range of white matter changes detected by MRI varied greatly but was always accompanied by ventricular distention, implying a global neurodegenerative process. Skin biopsy was not part of the evaluation, but presumably many or all of the 12 individuals have NIID. Though it will be important to assess other ethnic populations, the writing appears to be on the wall: NOTCH2NLC repeat expansion disease should be considered in the differential diagnosis of patients displaying a progressive leukoencephalopathy with cognitive impairment, neuropathy and autonomic dysfunction, with or without accompanying movement disorder or weakness.

The list of repeat expansion diseases continues to grow (see table) and the use of new methods that can rapidly pinpoint disease-linked simple tandem repeats $(9,10)$ suggests more likely will be discovered soon. As scientists explore the underlying mechanisms of various repeat expansion diseases, it has become increasingly clear that they cluster in groups sharing clinical and molecular features (11). Many features of NOTCH2NLC repeat-mediated disease indicate that it belongs to one such cluster: neurodegenerative disorders caused by GC-rich repeats residing in non-protein coding regions of the respective disease genes. Well-known members of this class include fragile X-associated tremor ataxia syndrome (FXTAS) and C9ORF72mediated frontotemporal dementia/amyotrophic lateral sclerosis (FTD/ALS). Indeed, it was the similarity of NIID to FXTAS, including characteristic white matter changes, that led investigators (5) to search for repeat expansions in NIID and two similar conditions, oculopharyngeal myopathy with leukoencephalopathy (OPML) and oculopharyngodistal myopathy (OPDM). State-of-the-art techniques uncovered separate GGC(CGG) repeat expansions in all three diseases: in the 5' UTR of NOTCH2NLC (NIID), 5' UTR of LRP12 (OPDM) and the bidirectionally transcribed long noncoding RNAs LOC642361 and NUTM2B-AS1 (OPML).

How do noncoding GC-rich repeats in NOTCH2NLC and these other new repeat expansion genes cause disease? The inheritance pattern in families with NIID favors a dominant gain-offunction mechanism. Moreover, the ubiquitin- and p62-positive inclusions found throughout the brain in NIID are reminiscent of similar inclusions in FXTAS and C9ORF72 FTD/ALS. Perhaps, as in these well-studied diseases (12), repeat associated non-ATG (RAN) translation across the GGC repeat generates aggregation-prone RAN proteins. A second nonexclusive possibility is that the repeat expansion sequesters RNA binding proteins, thereby disrupting RNA homeostasis. Stay tuned as studies of these newly identified repeat expansions test these hypotheses.

On a final note, white matter disturbances are a frequent finding in progressive dementing disorders. While late-life dementia often carries a strong neurovascular component, many patients have white matter changes that seem disproportionate to their known cerebrovascular risk factors. In the right setting, we already screen for NOTCH3 mutations when the radiographic appearance, clinical characteristics and family history suggest CADASIL. For most neurologists, however, NIID has not been on their radar. This new report suggests that 
screening for the NOTCH2NLC repeat expansion will aid in the genetic evaluation of agerelated cognitive impairment accompanied by leukoencephalopathy, especially (but not only) when there is a family history of similar disease.

This article is protected by copyright. All rights reserved. 


\section{References}

1. Kunii M, Doi H, Ishii Y, Ohba C, Tanaka K, et al. Genetic analysis of adult leukoencephalopathy patients using a custom-designed gene panel. Clin Genet. 2018;94:232238. DOI: 10.1111/cge.13371. Epub 2018 Jun 8. PubMed PMID: 29700822.

2. Lynch DS, Wade C, Paiva ARB, John N, Kinsella JA. Practical approach to the diagnosis of adult-onset leukodystrophies: an updated guide in the genomic era. J Neurol Neurosurg Psychiatry. 2019;90:543-554. doi: 10.1136/jnnp-2018-319481. PubMed Central PMCID: PMC6581077.

3. Okubo M, Doi H, Fukai R, Fujita A, Mitsuhashi S, et al. GGC repeat expansion of TCH2NLC in adult patients with leukoencephalopathy. Ann Neurol. 2019 Aug 21. doi: 0.1002/ana.25586. [Epub ahead of print] PubMed PMID: 31433517.

4. Sone J, Mitsuhashi S, Fujita A, Mizuguchi T, Hamanaka K. Long-read sequencing identifies GGC repeat expansions in NOTCH2NLC associated with neuronal intranuclear inclusion disease. Nat Genet. 2019;51:1215-1221. doi: 10.1038/s41588-019-0459-y. Epub 2019 Jul 22. PubMed PMID: 31332381.

5. Ishiura H, Shibata S, Yoshimura J, Suzuki Y, Qu, et al. Noncoding CGG repeat expansions in neuronal intranuclear inclusion disease, oculopharyngodistal myopathy and an overlapping disease. Nat Genet. 2019;51(8):1222-1232. doi:10.1038/s41588-019-0458-z. Epub 2019 Jul 22. PubMed PMID: 31332380

6. Tian Y, Wang JL, Huang W, Zeng S, Jiao B, et al. Expansion of Human-Specific GGC Repeat in Neuronal Intranuclear Inclusion Disease-Related Disorders. Am J Hum Genet. 2019;105:166-176. doi: 10.1016/j.ajhg.2019.05.013. PubMed Central PMCID: PMC6612530.

7. Deng J, Gu M, Miao Y, Yao S, Zhu M, et al. Long-read sequencing identified repeat expansions in the 5'UTR of the NOTCH2NLC gene from Chinese patients with neuronal intranuclear inclusion disease. J Med Genet. 2019. pii: jmedgenet-2019-106268. doi: 10.1136/jmedgenet-2019-106268. [Epub ahead of print] PubMed PMID: 31413119.

8. Sone J, Mori K, Inagaki T, Katsumata R, Takagi S, et al. Clinicopathological features of adult-onset neuronal intranuclear inclusion disease. Brain. 2016 Dec;139(Pt 12):3170-3186. PubMed Central PMCID: PMC5382941.

9. Doi K, Monjo T, Hoang PH, Yoshimura J, Yurino $\mathrm{H}$, et al. Rapid detection of expanded short tandem repeats in personal genomics using hybrid sequencing. Bioinformatics. 2014; 30(6):815-22. doi: 10.1093/bioinformatics/btt647. PubMed Central PMCID: PMC3957077.

10. Bahlo M, Bennett MF, Degorski P, Tankard RM, Delatycki MB, Lockhart PJ. Recent advances in the detection of repeat expansions with short-read next-generation sequencing. 
F1000Res. 2018;7. pii: F1000 Faculty Rev-736. doi: 10.12688/f1000research.13980.1. PubMed Central PMCID: PMC6008857.

11. Paulson H. Repeat expansion diseases. Handb Clin Neurol. 2018;147:105-123. doi: 10.1016/B978-0-444-63233-3.00009-9. PubMed Central PMCID: PMC6485936.

12. Rodriguez CM, Todd PK. New pathologic mechanisms in nucleotide repeat expansion disorders. Neurobiol Dis. 2019;130:104515. doi: 0.1016/j.nbd.2019.104515. PubMed Central PMCID: PMC6689435. 


\section{Repeat Expansions and Associated Neurologic Disorders}

CAG - at least 10 diseases (HD, SBMA, DRPLA, 7 SCAs)

CTG - myotonic dystrophy type 1, HD-like 2, SCA8, Fuchs corneal dystrophy

GAA - Friedreich ataxia

GCC, CCG - FRAXE mental retardation, other fragile sites (GCC, CCG)

CGG - fragile X syndrome, FXTAS

GCG - oculopharyngeal muscular dystrophy (OPMD)

CGG - OPML, OPDM

GGC/CGG - NIID, leukoencephalopathy

CCTG - myotonic dystrophy type 2

AAGGG - cerebellar ataxia, neuropathy, vestibular areflexia syndrome (CANVAS)

TTTCA - benign adult familial myoclonic epilepsies (BAFME 1, 6, and 7)

ATTCT - SCA 10

TGGAA - SCA 31

GGCCTG - SCA 36

GGGGCC - C9ORF72 FTD/ALS

CCCCGCCCCGCG - EPM1 myoclonic epilepsy

Microsatellite expansions of the indicated repeat sequences are associated with a wide range of neurological diseases. GGC/CGG expansions (bold type) were recently identified in NIID/leukoencephalopathy and two similar, rare neurodegenerative diseases, oculopharyngeal myopathy with leukoencephalopathy (OPML) and oculopharyngodistal myopathy (OPDM). Together with FXTAS, these diseases, which are all caused by noncoding expansions consisting only of $\mathrm{G}$ and $\mathrm{C}$, constitute a spectrum of related neurodegenerative disorders with shared clinical, radiographic and neuropathological features. Abbreviations: DRPLA, dentatorubral-pallidoluysian atrophy; FXTAS, fragile X-associated tremor ataxia syndrome; FTD/ALS, frontotemporal dementia/amyotrophic lateral sclerosis; HD, Huntington disease; NIID, neuronal intranuclear inclusion disease; OPML; SBMA, spinal bulbar muscular atrophy; SCA, spinocerebellar ataxia.

This article is protected by copyright. All rights reserved. 


\section{Repeat Expansions and Associated Neurologic Disorders}

CAG - at least 10 diseases (HD, SBMA, DRPLA, 7 SCAs)

CTG - myotonic dystrophy type 1, HD-like 2, SCA8, Fuchs corneal dystrophy

GAA - Friedreich ataxia

GCC, CCG - FRAXE mental retardation, other fragile sites (GCC, CCG)

CGG - fragile X syndrome, FXTAS

GCG - oculopharyngeal muscular dystrophy (OPMD)

CGG - OPML, OPDM

GGC/CGG - NIID, leukoencephalopathy

CCTG - myotonic dystrophy type 2

AAGGG - cerebellar ataxia, neuropathy, vestibular areflexia syndrome (CANVAS)

TTTCA - benign adult familial myoclonic epilepsies (BAFME 1, 6, and 7)

ATTCT - SCA 10

TGGAA - SCA 31

GGCCTG - SCA 36

GGGGCC - C9ORF72 FTD/ALS

CCCCGCCCCGCG - EPM1 myoclonic epilepsy

Microsatellite expansions of the indicated repeat sequences are associated with a wide range of neurological diseases. GGC/CGG expansions (bold type) were recently identified in NIID/leukoencephalopathy and two similar, rare neurodegenerative diseases, oculopharyngeal myopathy with leukoencephalopathy (OPML) and oculopharyngodistal myopathy (OPDM). Together with FXTAS, these diseases, which are all caused by noncoding expansions consisting only of $\mathrm{G}$ and $\mathrm{C}$, constitute a spectrum of related neurodegenerative disorders with shared clinical, radiographic and neuropathological features. Abbreviations: DRPLA, dentatorubral-pallidoluysian atrophy; FXTAS, fragile X-associated tremor ataxia syndrome; FTD/ALS, frontotemporal dementia/amyotrophic lateral sclerosis; HD, Huntington disease; NIID, neuronal intranuclear inclusion disease; OPML; SBMA, spinal bulbar muscular atrophy; SCA, spinocerebellar ataxia.

This article is protected by copyright. All rights reserved. 